\title{
Impaired osteoblast and osteoclast function characterize the osteoporosis of Snyder - Robinson syndrome
}

\author{
Jessica S Albert ${ }^{1,2}$, Nisan Bhattacharyya ${ }^{3}$, Lynne A Wolfe ${ }^{1,2}$, William P Bone ${ }^{1}$, Valerie Maduro ${ }^{1}$, John Accardi ${ }^{1}$, \\ David R Adams ${ }^{1,2}$, Charles E Schwartz ${ }^{4}$, Joy Norris ${ }^{3}$, Tim Wood ${ }^{4}$, Rachel I Gafni ${ }^{3}$, Michael T Collins ${ }^{3}$, Laura L Tosi ${ }^{5,6}$, \\ Thomas C Markello ${ }^{1,2}$, William A Gahl ${ }^{1,2^{*}}$ and Cornelius F Boerkoel ${ }^{1}$
}

\begin{abstract}
Background: Snyder-Robinson Syndrome (SRS) is an X-linked intellectual disability disorder also characterized by osteoporosis, scoliosis, and dysmorphic facial features. It is caused by mutations in SMS, a ubiquitously expressed gene encoding the polyamine biosynthetic enzyme spermine synthase. We hypothesized that the tissue specificity of SRS arises from differential sensitivity to spermidine toxicity or spermine deficiency.

Methods: We performed detailed clinical, endocrine, histopathologic, and morphometric studies on two affected brothers with a spermine synthase loss of function mutation (NM_004595.4:C.443A > G, p.Gln148Arg). We also measured spermine and spermidine levels in cultured human bone marrow stromal cells (hBMSCs) and fibroblasts using the Biochrom 30 polyamine protocol and assessed the osteogenic potential of hBMSCs.

Results: In addition to the known tissue-specific features of SRS, the propositi manifested retinal pigmentary changes, recurrent episodes of hyper- and hypoglycemia, nephrocalcinosis, renal cysts, and frequent respiratory infections. Bone histopathology and morphometry identified a profound depletion of osteoblasts and osteoclasts, absence of a trabecular meshwork, a low bone volume and a thin cortex. Comparison of cultured fibroblasts from affected and unaffected individuals showed relatively small changes in polyamine content, whereas comparison of cultured osteoblasts identified marked differences in spermidine and spermine content. Osteogenic differentiation of the SRS-derived hBMSCs identified a severe deficiency of calcium phosphate mineralization.
\end{abstract}

Conclusions: Our findings support the hypothesis that cell specific alterations in polyamine metabolism contribute to the tissue specificity of SRS features, and that the low bone density arises from a failure of mineralization.

Keywords: Spermine, Snyder-Robinson syndrome, Osteoblast, Osteoclast, Osteoporosis

\section{Background}

Polyamines are ubiquitous, aliphatic, positively charged molecules that interact with anionic compounds such as DNA, RNA, and ATP $[1,2]$. Homeostasis of the polyamines putrescine, spermidine, and spermine is essential to cell growth and survival [3]. By addition of a propylamine moiety, spermidine synthase (SRM) converts putrescine into spermidine, and spermine synthase (SMS)

\footnotetext{
* Correspondence: gahlw@helix.nih.gov

'Undiagnosed Diseases Program, Common Fund, Office of the Director,

National Institutes of Health, Bethesda, MD 20814, USA

${ }^{2}$ Medical Genetics Branch, National Human Genome Research Institute, Bethesda, MD, USA

Full list of author information is available at the end of the article
}

converts spermidine into spermine [4]. The balance of spermine and spermidine is crucial for ion channel regulation, transcription and translation [5-9].

Mutations of SMS, the gene encoding spermine synthase, cause Snyder-Robinson syndrome (SRS), an X-linked disorder first reported in 1969 [10]. The clinical features of SRS include intellectual disability, dysmorphic facies, speech and gait abnormalities, seizures, muscle hypoplasia, kyphoscoliosis, and osteoporosis [11-17]. All affected males have hemizygous mutations in $S M S$ that result in reduced SMS activity and a decreased spermine:spermidine ratio. 
Atraumatic osteoporotic fractures commonly occur in individuals with SRS, leading to significantly impaired quality of life. Osteoporosis arises from disruption of the equilibrium between osteoclastic bone resorption and osteoblastic bone formation [18], which is regulated by mechanical and endocrine stimuli [19]. This general understanding of osteoporosis has led to established therapeutic interventions, but further insights are required to address the osteoporosis of SRS in a disease-specific manner.

Here we define the osteoporotic disease of SRS in two brothers with a missense mutation in SMS [20] and report depletion of osteoblasts and osteoclasts, reduced cancellous and cortical bone, reduced calcium-phosphate mineralization in vitro, and markedly abnormal polyamine content in human bone marrow stromal cells (hBMSCs). These data offer new insights into the role of polyamines in bone formation.

\section{Clinical reports \\ Patient II-1}

The propositus (II-1, Figure 1) is the 18-year-old son of non-consanguineous healthy parents with no family history of intellectual disability or skeletal problems.

He was born by cesarean section at 40 weeks following a gestation complicated by poor maternal weight gain and, at 8 months, atypical fetal movements suggestive of in utero seizures. His birth weight, length and occipitofrontal circumference (OFC) were $2.38 \mathrm{~kg}(3 \%), 47 \mathrm{~cm}(13 \%)$ and $34.5 \mathrm{~cm}$ (26\%), respectively. Apgar scores were 8 and 9 at one and five minutes. He had saggy skin but no other dysmorphic features. Following a perinatal intraventricular hemorrhage associated with thrombocytopenia $\left(7 \times 10^{3}\right.$ cells $\left./ \mu \mathrm{l}\right)$ that corrected after 3 platelet transfusions, he developed seizures, apnea with cyanosis, temperature instability and hypoglycemia. His recurrent episodes of hyperglycemia and hypoglycemia resolved with age and the placement of a gastric tube that allowed more frequent feedings.

Patient II-1 had tracheomalacia, upper airway obstruction, increased respiratory secretions, frequent aspirations, and pulmonary infections from infancy. By age 7 years, he had chronic Pseudomonas aeruginosa respiratory infection. At 6 years, he developed proximal renal tubular acidosis (RTA), nephrocalcinosis and nephrolithiasis. His renal stones were composed of carbonate apatite and calcium oxalate. His RTA has been managed with fluid and electrolyte replacement, spironolactone and hydrochlorothiazide.

Although initially controlled with phenobarbital, his seizures progressed to infantile spasms by 15 months. Adrenocorticotropic hormone (ACTH) treatment transiently reduced seizure frequency. His current anticonvulsant therapy includes rufinamide, felbamate, clonazepam, and topiramate. He had delayed development with regression of several developmental milestones. He smiled and laughed by 4 months, reached for toys by 6 months, had vocalizations by 7 months, and could hold his head up and roll onto his side by 8 months. He achieved his pincer grasp at 18 months but lost it by 23 months. He never walked. He lost many motor skills and all vocalization by 26 months of age. A cranial MRI at 14 years revealed cystic encephalomalacia and a low parenchymal T2 signal in the right temporal and right occipital lobes. These findings were considered consequences of the perinatal intraventricular hemorrhage.

When first evaluated at NIH at 15 years, Patient II-1 was awake but not interactive and could not sit independently or hold up his head; he withdrew from noxious stimuli. His height, weight and OFC were $129 \mathrm{~cm}$ (<3\%ile), $30.8 \mathrm{~kg}(<3 \%$ ile) and $50.3 \mathrm{~cm}(<3 \%$ ile), respectively. Facial dysmorphisms included a long, oval, asymmetric face, midface hypoplasia, down-slanting palpebral fissures, large, cupped ears, smooth philtrum, higharched palate and prognathia (Figure 1). His dental enamel was hypoplastic and secondary teeth 2, 6, 10, and 11 were absent. He had excessive drooling, sluggish pupillary reflexes and left-sided hearing loss. He had frequent seizures, severe hypotonia, decreased muscle bulk, hypoactive deep tendon reflexes, kyphoscoliosis and flexion contractures of most large and small joints (Figure 1). He had one prepubertal $(1-2 \mathrm{~mL})$ testis that was undescended but palpable in the inguinal canal and one undescended testis, Tanner stage III pubic hair and a prepubertal phallus. His skin was remarkable for excretions of carbonate apatite, reflecting calcium/phosphate dysregulation. His ophthalmologic exam revealed retinitis pigmentosa and cortical blindness.

Previous skeletal problems included congenital bilateral hip dislocation and fractures of his distal fibula (2 years), right humerus ( 5 years) and spine ( 6 years). Kyphoscoliosis developed between 6 and 12 years of age. A dual-energy $\mathrm{x}$-ray absorptiometry (DEXA) scan performed at age 15 years demonstrated a bone density of $0.341 \mathrm{gm} / \mathrm{cm}^{2} \mathrm{BMD}$ (height adjusted Z-Score: -2.9 [21]) for the anteroposterior Spine (L1-L4) and $0.342 \mathrm{gm} / \mathrm{cm}^{2}$ bone mineral density (height adjusted Z-Score: -6.5 [21]) for the right forearm. Skeletal radiographs at 18 years revealed a $60^{\circ}$ convex right scoliosis, gracile bones with reduced mineral density, and evidence of previous fractures (Figure 2E-J).

\section{Patient II-3}

Patient II-3, the brother of Patient II-1, was born at 37 weeks by cesarean section following an uncomplicated pregnancy. His birth weight was $2.81 \mathrm{~kg}$ (25\%). During the immediate neonatal period his platelet count decreased from 90 to $45 \mathrm{k} / \mu \mathrm{L}$, and he was admitted to the neonatal intensive care unit for treatment with 

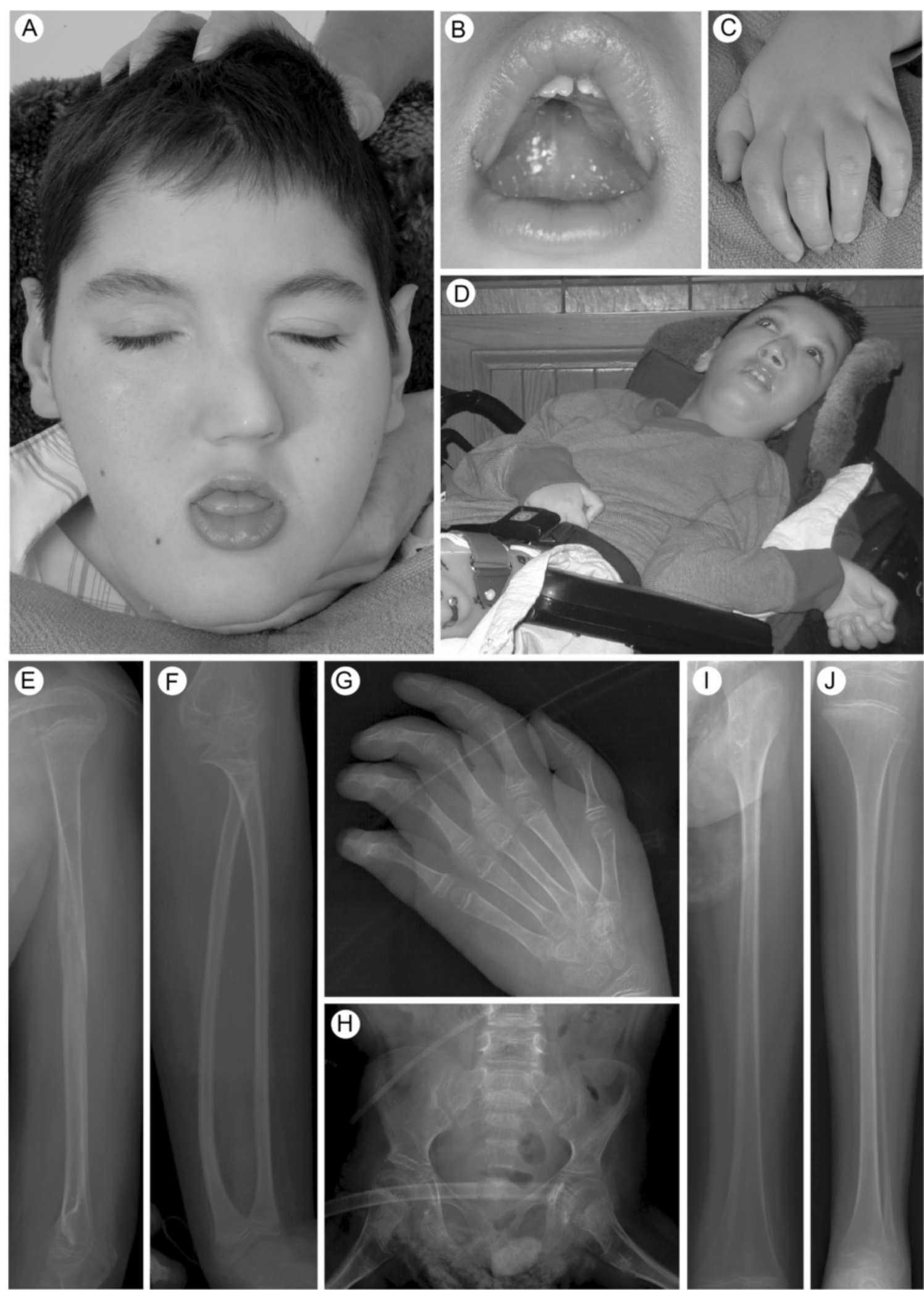

Figure 1 Clinical and radiographic features. A. Face of patient II-1. B. Face of patient II-3. C. Palate of patient II-1. D. Hands of patient II-3. E-J. Skeletal radiographs of Patient II-1 showing the left humerus (E), left forearm (F), left hand (G), pelvis (H), left femur (I) and left lower leg (J). Note the gracile bones and undermineralization as well as the healing humeral fracture (E). 
A

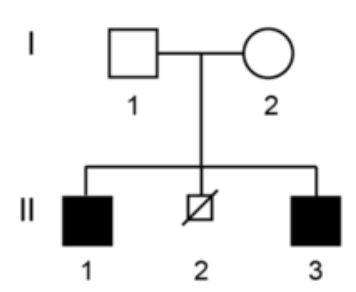

B

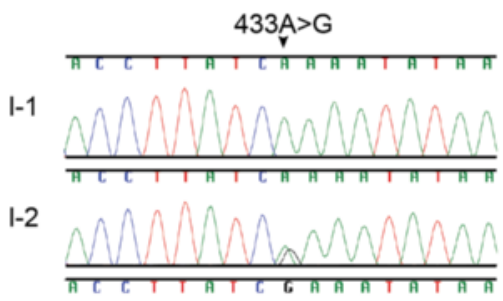

II-1

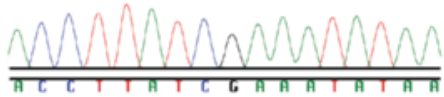

II-3

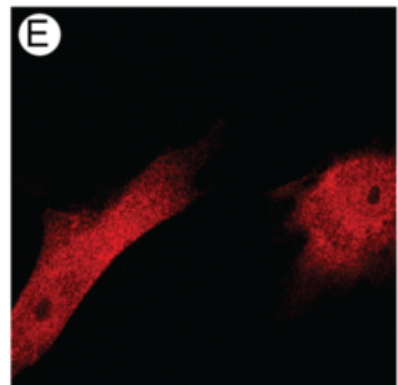

$\boldsymbol{F}$

Cnt II-1 II-3

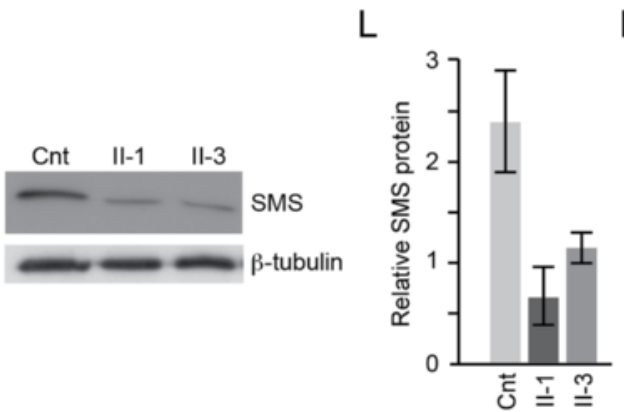

K

C

D

(H)

\section{Q148R}

H. sapiens 141 YEKDSAYQNIKILIHS

M. musculus 141 YDEDSPYQNIKILHS

D. rerio 138 YEKDSAYQNIKILHS

D. melanogaster 146 FEARSPFQKIQIMHS

S. cerevisiae 37 YEARSEFQDILIFRN
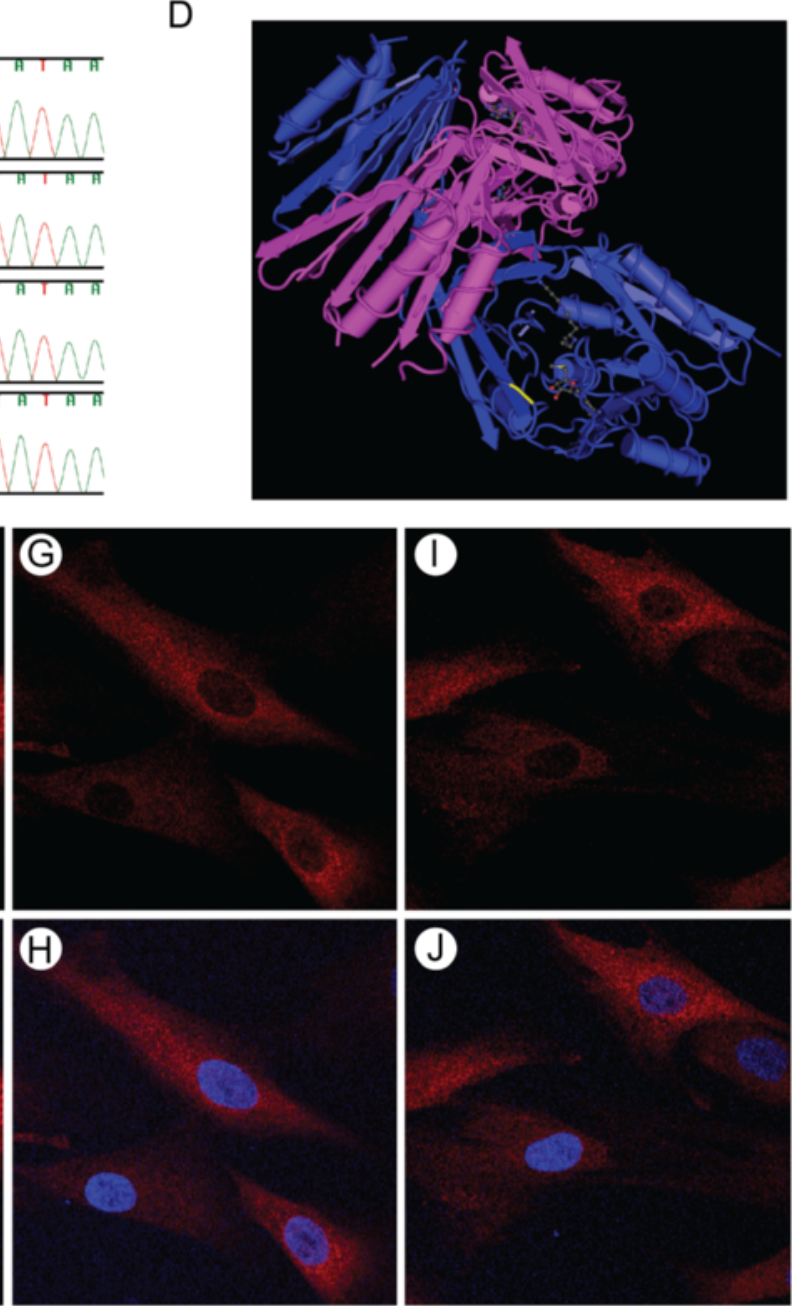

$\mathrm{N}$

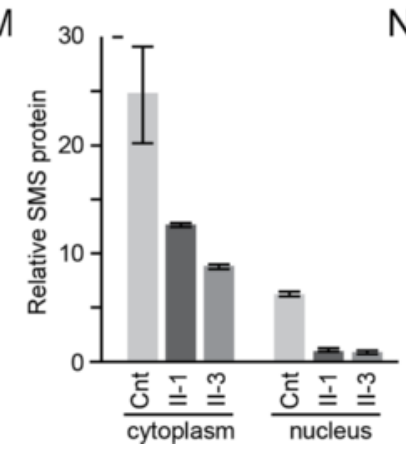

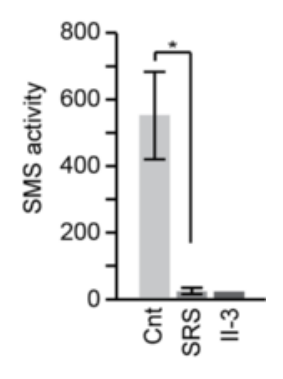

Figure 2 (See legend on next page.) 
(See figure on previous page.)

Figure 2 Segregation, mutational analysis, and functional consequences of a novel SMS variant. A. Pedigree of the family of the propositi. Affected males are shown by black squares. B. Sanger sequencing chromatograms showing the segregation of the SMS mutation NM_004595.4:c.443A $>\mathrm{G}$ from the carrier mother to the affected boys. The unaffected father did not have this mutation. C. Conservation of the p.GIn148 (p.Q148) residue across species. D. Drawing of the human SMS protein crystal complexed with spermidine and 5-methylthioadenosine. The mutated amino acid (Gln 148) is highlighted in yellow [Mac PyMOL [23]]. E-J. Immunofluorescent detection of SMS protein subcellular distribution in unaffected (E, F), Patient II-1 (G, H) and Patient II-3 (I, J) skin fibroblasts. SMS protein is shown in red and the nucleus is shown in blue. $\mathbf{K}$. Immunoblot of skin fibroblast lysates showing reduced SMS protein levels in the patients (II-1, II-3) compared to an unaffected control (cnt). Tubulin is shown as a loading control. L. Graph showing steady state SMS protein levels in the patient and control fibroblasts relative to ß-tubulin levels. The data are based on 3 independent experiments for each cell line. $\mathbf{M}$. Graph quantifying immunoblot detected steady state SMS protein levels in the cytoplasm and nuclei of patient and control fibroblasts. The cytoplasmic expression was normalized to $\beta$-tubulin expression and the nuclear expression to p84 expression. The data are based on 2 independent experiments for each cell line. N. SMS enzyme activity (spermidine d8 peak per hour) in lymphoblasts of unaffected individuals (Cnt), a cohort of 4 individuals with SRS (SRS) and patient II-1, ${ }^{*} \mathrm{p}<0.05$.

dexamethasone and a platelet transfusion. He also had transient hypoglycemia, poor feeding and sensitivity to light. By 4 days of life, his condition had stabilized and he was discharged. Over the subsequent months, he manifested moderate laryngomalacia, mild tracheobrochomalacia, severe torticollis and abnormally pigmented retinas. Like his brother, he has also had episodic hyper- and hypoglycemia. He was diagnosed with nephrocalcinosis at 1 year and RTA by 2 years. The composition of his renal stones was carbonate apatite and calcium oxalate.

Patient II-3 had global developmental delay, severe hypotonia, and regression of milestones; he lost vocalizations and most motor skills by 15 months. An EEG at 6 months showed generalized slowing and disorganization; at 18 months he manifested seizures and hypsarrhythmia. An MRI performed at 2 years of age showed a mild increase in ventricular size but no other abnormalities. There was no evidence of hemorrhage or malformation. Since age 5 years, he has required a vagal nerve stimulator and bi-pap for adequate respiration. He also had repeated Pseudomonas aeruginosa pulmonary infections. His infections are more frequent and severe than are those of his brother.

Patient II-3 has had multiple atraumatic fractures involving the clavicle, tibia, femur and humerus. He also had congenital bilateral hip dislocation and, by 4 years of age scoliosis.

Illness prevented Patient II-3 from traveling to the $\mathrm{NIH}$ for evaluation, but review of his medical records and photographs showed that he was alert and nonambulatory at age 10 years. He had facial features similar to those of his brother (Figure 1), superficial skin excretions, hypotonia, large joint contractures and muscle atrophy.

\section{Laboratory studies}

The propositi had extensive laboratory testing. This identified an elevated antibody titer to myelin basic protein and a mild intermittent anemia with low iron saturation (7\%; normal, 15-62) and ferritin (20 mcg/L; normal,
26 -388) for Patient II-1 and an elevated blood lactate level of $7.8 \mathrm{mmol} / \mathrm{L}(0.5-2.2)$ and mild elevations of urine carnitine esters for Patient II-3. Testing for abnormalities in organic acids, amino acids, acylcarnitines, very long chain fatty acids, lysosomal enzymes, biotinidase and copper were unremarkable. Molecular testing of $M E C P 2$, mitochondrial DNA, a panel of lysosomal storage diseaseassociated genes and the X-Linked Mental Retardation 9 Gene Panel (Greenwood Clinic, 2008), which did not include SMS and FRMPD4, did not detect pathogenic mutations. Patient II-1 had a normal karyotype (46,XY), and clinical and research copy number variant analysis did not detect any pathogenic variants (Additional file 1: Table S1).

\section{Methods \\ Patients}

The propositi were accepted into the NIH Undiagnosed Diseases Program (UDP) and enrolled in clinical protocol 76-HG-0238, approved by the Institutional Review Board of the National Human Genome Research Institute. Their parents gave written, informed consent.

\section{CNV analysis}

The NHGRI Genomics Core lab performed SNP determinations using the Illumina Bead Array Platform (HumanOmniExpress, Illumina Corp., San Diego, CA, USA). Genomewide fluorescent intensities and genotype calls were analyzed using Bead Studio and Genome Studio (Illumina Corp.). Analysis of copy-number variations was performed using PennCNV software, [22] and visual inspection using Genome Studio version 2010v3 build37/hg19 [23].

\section{Exome sequence analysis}

Genomic DNA was extracted from whole blood using the Gentra Puregene Blood kit (Qiagen, Valencia, CA) according to the manufacturer's specifications. Exome sequencing and analysis were performed as described [24-26]. The potential pathogenicity of identified variants was predicted using CDPred, SIFT, PolyPhen2 [27-33] or the BLOSUM62 scoring matrix [34]. 


\section{Confirmatory sequencing}

For the SMS variant, Qiagen HotStarTaq master mix (Qiagen, Valencia, CA) was used to amplify the putative variant and 200 flanking nucleotides using the primers $5^{\prime}$ TGTGGCTTTCTTTTGCACAC-3' and 5'-TGCATCT CAAAAACCAGCAG-3'. Unincorporated primers and nucleotides were removed using ExoSAP-IT reagent (USB, Cleveland, OH, USA). Sanger capillary sequencing was used to sequence the PCR products (Macrogen, Rockville, MD), and the sequences were aligned and analyzed using Sequencher v.4.10.1 (Gene Codes, Ann Arbor, MI, USA). Mutation interpretation was conducted using Alamut 2.0 (Interactive Biosoftware, San Diego, CA, USA).

\section{Bone biopsy and histomorphometry}

Patient II-1 was given two courses of demeclocycline prior to the biopsy so that dynamic histomorphometry, including measurement of bone turnover, could be performed [35]. A bicortical transiliac crest core biopsy was performed and immediately split. The sample was placed in $70 \%$ ethanol and sent to The Johns Hopkins School of Medicine for histomorphometry, which was performed as described [35].

\section{Western blot analysis}

Protein was extracted from fibroblast and osteoblast cell lines with RIPA buffer (Thermo Scientific, Waltham, MA) or used directly from Clontech human protein library (Clontech Laboratories, Mountain View, CA). Lysates (30ug) were electrophoresed on a 4-10\% SDS-polyacrylamide gel and transferred to a polyvinylidene fluoride (PVDF) membrane. Using gentle agitation, the membrane was blocked overnight at $4^{\circ} \mathrm{C}$ with casein blocking buffer (Thermo Scientific, Waltham, MA) and 10\% horse serum. Anti-SMS (1:1000) (Novus Biologicals, Littleton, CO), anti- $\beta$-tubulin (1:1000, AbCam, Cambridge, UK), and anti-GAPDH (1:1000, Gene Tex, Irvine, CA) were used as primary antibodies. Horseradish peroxidase-conjugated secondary antibodies (1:10000, Bio-rad Laboratories, Hurcules, CA) were used to detect the primary antibodies. The antibody-enzyme complexes were detected by chemiluminescence using Amersham ECL western blotting detection reagent (GE Life Sciences, Pittsburgh, PA) or WesternSure Premium Chemiluminescent Substrate (LI-COR, Lincoln, NE) according to the manufacturer's specifications. $\beta$-tubulin or GAPDH was detected as a loading control.

\section{Cellular fractionation}

Cellular fractionation was performed using NE-PER Nuclear and Cytoplasmic Extraction Reagents (Thermo Scientific, Waltham, MA) per the manufacturer's recommendations. Immunoblotting was performed as described above. $\beta$-tubulin and p84 (GeneTex, Irvine, CA) served as loading controls for the cytoplasmic and nuclear fractions, respectively.

\section{qRT-PCR analysis}

RNA was extracted from cells using the RNeasy Mini Kit (Qiagen, Valencia, CA) per the manufacturer's specifications. RNA was converted to cDNA using the VILO cDNA synthesis kit (Life Technologies, Grand Island, NY) per the manufacturer's protocol. 100ng of cDNA was amplified using Sso Advanced SYBR Green supermix (Bio-Rad Laboratories, Richmond, CA) per the manufacturer's specification. qRT-PCR for measuring SMS steady state mRNA levels was performed on the Bio-Rad CFX96 Real-Time system (Bio-Rad Laboratories, Richmond, CA) using primers $5^{\prime}$-gattggtgttgctggacctt-3' and $5^{\prime}$-tgactcaattcttt cattcttcct-3'. PCR was cycled 33 times and annealing temperature was 58 degrees, melt curve was incremented at 0.5 degrees from 65-95 degrees. mRNA levels were normalized to GAPDH mRNA levels, a house-keeping gene.

\section{Cell culture}

Epstein-Barr virus (EBV)-transformed lymphoblast cells were cultured as described previously [36]. Fibroblast cells were cultured in Dulbecco's Modified Eagle Medium (DMEM) supplemented with $4.5 \mathrm{~g} / \mathrm{L}$ D-glucose, L-glutamine, sodium pyruvate (Life Technologies, Grand Island, NY), 10\% fetal bovine serum (Life Technologies, Grand Island, NY) and $1 \mathrm{X}$ antibiotic (Life Technologies, Grand Island, NY). Fibroblasts were grown at $37^{\circ} \mathrm{C}$ with $5 \% \mathrm{CO} 2$.

Human bone marrow stromal cells (hBMSC) were isolated and grown in culture as previously described [37]. Briefly, bone biopsy samples from patient(s) were used as the starting material. Cells were grown in $\alpha$-minimal essential medium ( $\alpha$-MEM) containing 20\% fetal bovine serum (FBS, Atlanta Biologicals, Lawrenceville, GA, USA), L-glutamine (Glutamax, GIBCO, Carlsbad, CA, USA), and penicillin-streptomycin mix. When noted, cells were also grown in the above medium containing Dexamethasone (10-8 M dexamethasone and 10-4 M ascorbate). Cells were cryopreserved in $\alpha$-MEM containing 50\% FBS and $5 \%$ dimethyl sulphoxide (DMSO). Control hBMSC were obtained from the rib of a 51 year-old Caucasian male.

\section{Osteogenic differentiation assay}

Control and experimental BMSCs were plated (6x104/12well plate) in triplicate. Cells were kept either untreated ( $\alpha$ MEM containing 20\% FBS, L-glutamine, antibiotics) or treated with an osteogenic differentiation media ( $\alpha$-MEM, $20 \%$ FBS, L-glutamine, antibiotics, 5x10-3M $\beta$-glycerophosphate, 1x10-4M Ascorbic Acid Phosphate and 1x10-8M dexamethasone). Media was changed every 3-4 days. After 18 days, cells were rinsed with HBSS (Hanks' Balanced Salt Solution, Invitrogen, Grand Island, NY) and were fixed at room temperature using $4 \%$ paraformaldehyde solution in 1X phosphate buffered saline (PBS). Cells were washed with distilled water, and were stained with Alizarin Red solution at room temperature for 20 minutes. Finally, the cells 
were washed with distilled water for 6 times and were photographed.

\section{Spermine synthase activity assay}

To test SMS activity in lymphoblastoid cell lines, we measured the production of deuterated spermine (spermine d8) from deuterated spermidine (spermidine d8). Briefly, cells were harvested by centrifugation and washed 2X with $\mathrm{PBS}$ and suspended in $50 \mathrm{mM}$ sodium phosphate buffer pH 7.2 with protease inhibitor (Sigma, St. Louis, MO). Samples were frozen at $-80^{\circ} \mathrm{C}$. Upon thawing, the samples were subjected to 2 freeze-thaw cycles using an ethanol and dry ice bath. After centrifugation, protein in the supernatant was quantified using the Lowry assay. To test for SMS activity, $70 \mu \mathrm{g}$ of protein was incubated with $0.1 \mathrm{M}$ sodium phosphate buffer 7.5 , $10 \mu \mathrm{M}$ spermidine d8 (Sigma, St. Louis, MO), protease inhibitor (Sigma, St. Louis, MO), 100 $\mu \mathrm{M}$ dcSAM, and 50uM 4-MCHA (Sigma, St. Louis, MO) in a total volume of $100 \mu \mathrm{L}$. Baseline reactions were stopped immediately with $100 \mu \mathrm{L}$ acetonitrile $/ 0.1 \%$ formic acid; other samples were incubated at $37^{\circ} \mathrm{C}$ for $24 \mathrm{~h}$ and then stopped by addition of acetonitrile $/ 0.1 \%$ formic acid. Spermine d8 was quantified by LC/MS/MS. Enzymatic activity was represented as the area of the spermidine $\mathrm{d} 8$ peak per hour.

\section{Measurement of polyamine content}

The spermine/spermidine ratio was determined in the lymphoblastoid cell lines using LC/MS/MS as described [36]. Fibroblast and hBMSC polyamines were measured using the Biochrom 30 polyamine protocol. Briefly, human fibroblasts and hBMSCs were cultured as described above. Cells were harvested and pelleted and polyamines were extracted with a volume of $10 \%$ PCA equivalent to 4-fold the weight of the cells in milligrams. After $1 \mathrm{~h}$ of incubation on ice, cells were centrifuged at 13,000 rpm for $10 \mathrm{~min}$ at 4 degrees. The polyamines were fractionated and quantitated on the Biochrom 30 using an ion exchange polyamine column, compatible with sodium chemistry.

\section{Immunofluorescent localization of SMS}

Immunofluorescent detection of SMS was modified from a previously described protocol [38]. Briefly, 2 x 104 cells were grown overnight on a coverslip in a 6-well plate. The cells were fixed with $3.7 \%$ paraformaldehyde (PFA) for 25 min at room temperature and permeabilized with $0.1 \%$ Triton X$100,2 \mathrm{mg} / \mathrm{ml} \mathrm{BSA}$ and $1 \mathrm{mM} \mathrm{NaN3}$ for $5 \mathrm{~min}$ at room temperature. All cells were blocked for $2 \mathrm{~h}$ with casein blocking buffer with $10 \%$ horse serum and then incubated at $4^{\circ} \mathrm{C}$ overnight with anti-SMS (1:100) (Sigma, St. Louis, $\mathrm{MO}$ ) diluted in blocking buffer. They were then gently washed 3 times with PBS and incubated with secondary antibodies conjugated with Alexa 488 or Alexa 555 (2ug/ml, Life Technologies, Grand Island, NY) for $2 \mathrm{~h}$ at room temperature. Cells were then washed 3 times with PBS and mounted in Vectashield containing 4', 6-diamidino-2-phenylindole (DAPI, Vector Laboratories, Burlington, ON, Canada). Images were acquired using a 63X Zeiss plan-apochromat oil, 1.4 NA, DIC objective lens on a Zeiss LSM 780 confocal microscope using Zen 2011 acquisition software.

\section{Alkaline phosphatase activity}

Upon reaching confluence, hBMSCs were plated in 6- well plates $(100,000$ cells/well). After 10 days of growth in MEM Alpha with 20\% FBS, the hBMSCs were stimulated with $10 \mathrm{mM} \beta$-glycerophosphate (Sigma St. Louis, MO), $50 \mu \mathrm{M}$ ascorbic acid 2-phosphate (Sigma, St. Louis, MO) and 100nM Dexamethasone (Sigma, St. Louis, MO) for 5 days. Alkaline phosphatase activity was quantified using StemTAG Alkaline Phosphatase Colorimetric Kit (Cell Biolabs, San Diego, CA).

\section{Results}

\section{Exome sequencing identifies a novel SMS mutation diagnostic of SRS}

The propositi had a maternally inherited hemizygous transition (NM_004595.4:c.443A > G) in SMS identified by exome sequencing (Additional file 1: Table S2; full VCF file is available on request) and confirmed by Sanger sequencing (Figure 2A-B). This mutation encodes the missense mutation p.Gln148Arg (CDPred score: -9; SIFT score: 0; PolyPhen2: probably damaging (0.998, Sensitivity 0.27, Specificity 0.99)). The Gln148 residue is conserved to $S$. cerevisiae (Figure $2 \mathrm{C}$ ) and resides in the central $\beta$-strand domain that functions as a cap for the carboxyl terminal catalytic domain. It is one of 8 residues involved in the binding of 5'-methylthioadenosine (MTA) (Figure 2D) [39,40], which functions as an amine acceptor.

Similar to other SRS-associated SMS mutations [11-17], the p.Gln148Arg variant decreased the steady state level of SMS protein detectable in cultured fibroblasts by immunofluorescence and immunoblotting (Figure 2E-K). It reduced total SMS steady state levels 2.6-fold (Figure 2L); nuclear SMS was reduced 5.8-fold and soluble cytosolic SMS 2.7fold (Figure 2M).

Spermine $\mathrm{d} 8$ generation by lymphoblastoid lysates expressing p.Gln148Arg SMS was 37-fold less than that for unaffected controls (Figure 2N). Additionally, as measured by LC/MS/MS, the ratio of spermine: spermidine in the lymphoblastoid lysates was reduced 10-fold compared to unaffected controls.

The propositi had features previously not reported with SRS, including more profound intellectual disability, retinal pigmentary changes, renal dysfunction, frequent pulmonary infections and hyper- and hypoglycemia (Table 1). Although 
Table 1 Comparison of clinical features of all reported SRS patients

\begin{tabular}{|c|c|c|c|}
\hline Features & $\begin{array}{l}\text { Reported } \\
\text { patients }\end{array}$ & Patient II-1 & Patient II-3 \\
\hline Cognitive impairment & $15 / 15$ & + & + \\
\hline Seizures & $8 / 15$ & + & + \\
\hline Myopia & $2 / 13$ & $+*$ & $+*$ \\
\hline High, narrow palate & $3 / 13$ & + & + \\
\hline Prominent lower lip & $12 / 15$ & + & - \\
\hline Speech abnormalities & $15 / 15$ & $+* *$ & $+* *$ \\
\hline Diminished body bulk & $15 / 15$ & + & + \\
\hline Kyphoscoliosis & 13/15 & + & + \\
\hline Osteoporosis & $11 / 11$ & + & + \\
\hline Long finger/toes & $12 / 15$ & $+* * *$ & $\#$ \\
\hline Unsteady gait & $10 / 15$ & $+* * * *$ & $+* * * *$ \\
\hline Renal abnormalities & $3 / 13$ & + & + \\
\hline Nephrocalcinosis $^{a}$ & $0 / 13$ & + & + \\
\hline Frequent infections & $0 / 13$ & + & + \\
\hline Retinal pigment changes & $0 / 13$ & + & + \\
\hline Hypo-/Hyper-glycemia & $1 / 13$ & + & + \\
\hline $\begin{array}{l}\text { Muscle fiber } \\
\text { abnormalities }\end{array}$ & $1 / 13$ & + & + \\
\hline
\end{tabular}

\#Not assessed.

*Partial blindness.

**No vocalizations.

${ }^{* * *}$ Contractures of fingers.

****Non-ambulatory.

${ }^{a}$ Note: Nephrocalcinosis in these patients previously recorded in GeneReviews [20].

The phenotype data used to generate this table was collected from [11-17].

we hypothesized that rare mutations in other genes contributed to these features, only a maternally inherited $\mathrm{X}$-linked variant in FRMPD4 (c.583A > G, p.K195E) segregated with the disease and had a predicted pathogenicity of at least probably damaging in Polyphen [27]; SIFT and CDPred predict the variant as tolerated. FRMPD4, which has been associated with autism and schizophrenia [41], encodes a product that regulates dendritic spine morphogenesis [42]. No pathogenic mutations were observed in genes associated with retinal pigmentary changes.

\section{Bone formation is decreased in patient II-1}

$S M S$ is widely expressed (Additional file 1: Figure S1); consequently, we questioned why specific tissues such as bone are particularly affected in SRS patients. To define better the osteoporosis of SRS, we performed a bicortical transiliac crest core biopsy of Patient II-1. The specimen was soft and fragmented when removed from the trephine (Figure 3A). Histomorphometry revealed an absence of a trabecular meshwork, a low bone volume and a thin cortex. Cancellous bone volume was markedly decreased at $4.7 \%$, compared with $23 \% \pm 4.4$ in healthy controls. The cortical mean width measured only 238 microns compared to a mean of 1202 microns in controls. Osteoblastic activity was markedly reduced with osteoblasts occupying only $1 \%$ of the osteoid surface (normal range $12.1 \% \pm 4.6$ ). There was no observable osteoclastic activity in the patient's specimen, demonstrated by an eroded surface of $0 \%$ (normal range $4.1 \% \pm 2.3$ ) and an osteoclast surface of $0 \%$ (normal range $0.7 \% \pm 0.6)$. Surface bone formation rate was $11.6 \mathrm{~m}^{3} / \mu \mathrm{m}^{2} / \mathrm{y}$ (normal range: $35.8 \mu^{3} / \mu \mathrm{m}^{2} / \mathrm{y} \pm 8.9$ ).

\section{CSF neurotransmitter levels do not suggest sympathetic} nervous system dysfunction in Patient II-1

Because bone density and turnover are controlled in part by the sympathetic nervous system [43-49], we measured CSF neurotransmitters in Patient II-1 for evidence of increased sympathetic activity. The 5-methyltetrahydrofolate (methyl donor), 5-hydroxyindoleacetic acid (serotonin metabolite), 3-O-methyldopa (metabolite of L-Dopa), and tetrahydrobiopterin (cofactor for the synthesis of serotonin, dopamine, norepinephrine) were all within normal limits. Although homovanillic acid (catecholamine metabolite) (459 nmol/L) and neopterin (catabolic product of GTP) (30 nmol/L) were slightly above normal limits ( 324 and 28 $\mathrm{nmol} / \mathrm{L}$, respectively), these slight elevations were considered clinically insignificant.

\section{Endocrine evaluation in patient II-1}

Because bone density is also modulated by multiple endocrine regulators [19], we assessed these in Patient II-1. Intact PTH, Vitamin D, magnesium, cortisol, insulinlike growth factor 1, thyroxine, thyroid stimulating hormone, prolactin and ACTH were unremarkable (Table 2). Also, manual inspection of the exome results did not identify any predicted deleterious mutations of IGF1 or IGF1R despite $>20$ fold depth of short read coverage across the length of each exon. Prior to admission to the $\mathrm{NIH}$, patients II-1 and II-3 had fluctuations in blood calcium and phosphate levels (Table 3). These fluctuations had no apparent pattern or mediating factor based on diet, fluid intake, or clinical status, and at the time of admission to $\mathrm{NIH}$, the blood levels of calcium and phosphorus were normal. Tubular reabsorption of phosphate was normal, but, the calculated 24-hr urine calcium excretion (derived from an 18-hr collection) was elevated. Osteocalcin levels were low for pubertal stage, consistent with decreased bone formation. At age 15 years, patient II- 1 had undescended testes, remarkably delayed puberty, low LH and FSH levels and undetectable testosterone. By age 18 years, he had entered early puberty as evidenced by an increase in LH and FSH, and testosterone levels in the Tanner II range. Testicular ultrasound at that time identified both testes in the inguinal canal, with volumes of $5.7 \mathrm{~mL}$ and 1.7 $\mathrm{mL}$. At a chronological age of 18 years and 4 months, his hone age was 12 years and 6 months. 


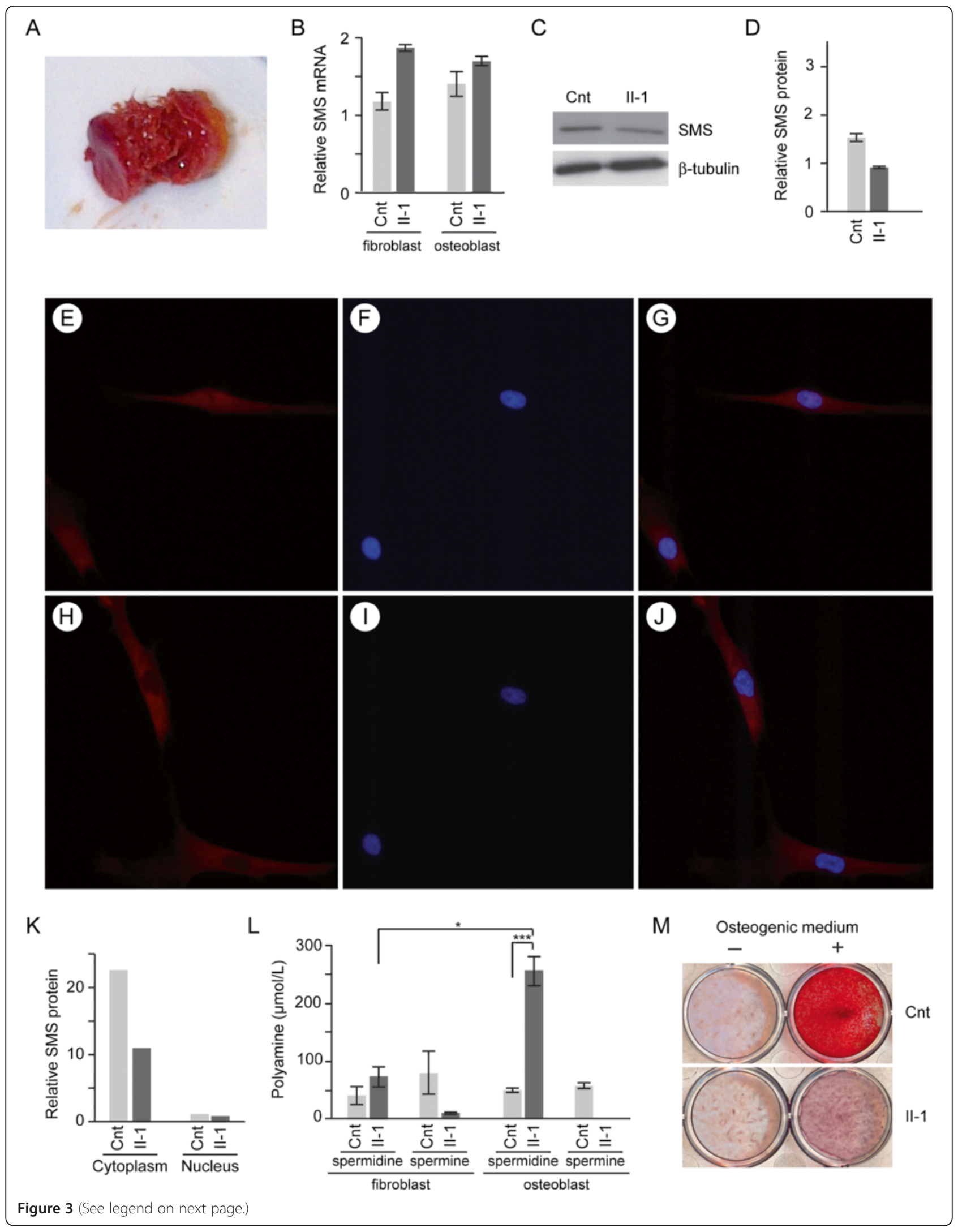


(See figure on previous page.)

Figure $\mathbf{3}$ Characterization of the bone and osteoblast pathology. A. Photograph of the bone biopsy. B. Steady state SMS mRNA levels relative to GAPDH expression in cultured fibroblasts and osteoblasts. The patient's cells did not differ significantly from controls. Data were derived by qRT-PCR analysis of 3 independent extractions of total RNA. C. Immunoblot showing steady state SMS protein expression in patient and control osteoblasts. B-tubulin is shown as a loading control. D. Graph showing steady state SMS protein levels in the patient and control hBMSCs relative to $ß$-tubulin levels; there was no significant difference. The data are based on 3 independent experiments for each cell line. E-J. Immunofluorescent detection of SMS protein subcellular distribution in unaffected (E-G) and Patient II-1 (H-J) hBMSCs. SMS protein is shown in red and the nucleus is shown in blue. $\mathbf{K}$. Graph quantifying immunoblot detected steady state SMS protein levels in the cytoplasm and nuclei of patient and control hBMSCs. The cytoplasmic expression was normalized to $\beta$-tubulin expression and the nuclear expression to p84 expression. L. Polyamine quantification in fibroblasts and osteoblasts. Note that the patient hBMSCs have a more striking imbalance of spermidine and spermine levels than do the patient fibroblasts, ${ }^{*} p<0.05,{ }^{* * *} p<0.005$. M. Osteogenic potential of bone marrow stromal cells (hBMSCs) isolated from Patient II-1 sample is markedly lower than that of an unaffected control (cnt). The hBMSCs were seeded in triplicates (6x10 $4 / 12$-well) and either kept untreated (-) or treated (+) with osteogenic differentiation media (see Methods) for 18 days. After the treatment, cells were fixed and were stained with Alizarin Red $\mathrm{S}$ to check for calcium deposition, a marker of osteogenic differentiation.

\section{Compared to cultured SRS fibroblasts, SRS hBMSC have} comparable SMS mRNA and protein levels but pronounced disturbances of polyamine levels

While absolute polyamine levels are not substantially abnormal in cultured fibroblasts and lymphoblastoid cells of SRS individuals, spermidine/spermine ratios reflected the SMS enzyme deficiency [12-17]. Neither lymphoblastoid cells nor skin fibroblasts, however, are affected tissues

Table 2 Markers of bone and endocrine function in Patient II-1 at 18 years of age

\begin{tabular}{|c|c|c|}
\hline Metabolite & $\begin{array}{l}\text { Patient II-1 } \\
\text { values }\end{array}$ & $\begin{array}{l}\text { Reference } \\
\text { range }\end{array}$ \\
\hline Intact parathyroid hormone $(\mathrm{pg} / \mathrm{mL})$ & 28.9 & $(15-65)$ \\
\hline 1,25-dihydroxycholecalciferol (pg/mL) & 70 & $(18-64)$ \\
\hline 25-dihydroxycholecalciferol (ng/mL) & 39 & $(33-100)$ \\
\hline Osteocalcin (ng/mL) & 35.7 & $\begin{array}{l}(7.3-38.5)^{1} \\
(49-167)^{2}\end{array}$ \\
\hline Free thyroxine (ng/dL, direct dialysis) & 1.6 & $(1-2.4)$ \\
\hline Thyroid stimulating hormone $(\mu \mathrm{l} \mathrm{U} / \mathrm{mL})$ & 1.8 & $(0.4-4)$ \\
\hline Testosterone (ng/mL) & 43.6 & $\begin{array}{l}(100-740)^{1} \\
(8-418)^{2}\end{array}$ \\
\hline Free testosterone (ng/dL) & 0.5 & $(7.4-22.6)^{1}$ \\
\hline $\begin{array}{l}\text { Sex hormone binding } \\
\text { globulin (nmol/L) }\end{array}$ & 63 & $\begin{array}{l}(10-60)^{1} \\
(44-160)^{2}\end{array}$ \\
\hline Follicle stimulating hormone (U/L) & 8.7 & $(1-11)^{1}$ \\
\hline Luteinizing hormone (U/L) & 2.5 & $(1-8)^{1}$ \\
\hline Prolactin (mcg/dL) & 9.4 & $(2-25)$ \\
\hline Cortisol (mcg/dL, morning) & 16.5 & $(5-25)$ \\
\hline Adrenocorticotropic hormone $(\mathrm{pg} / \mathrm{mL})$ & 12.7 & $(0.0-46.0)$ \\
\hline Insulin-like growth factor (ng/mL) & 347 & $(75-420)^{2}$ \\
\hline $\mathrm{pH}$ Urine & 8.5 & $(5-8)$ \\
\hline 18-hr urine volume (mL/24 h) & 2885 & $(600-1800)$ \\
\hline $\begin{array}{l}\text { Calculated urine calcium excretion } \\
(\mathrm{mmol} / \mathrm{kg} / 24 \mathrm{~h})\end{array}$ & 0.134 & $(<0.1)$ \\
\hline $\begin{array}{l}\text { Tubular reabsorption of } \\
\text { phosphorus (\%TRP) }\end{array}$ & 93.5 & $(85-95)$ \\
\hline
\end{tabular}

${ }^{1}$ Reference ranges for age, ${ }^{2}$ Reference range for pubertal stage. in SRS. We hypothesized therefore that cells from affected tissues such as bone had differences in SMS expression or polyamine metabolism accounting for their clinical manifestations. To test this, we compared SMS expression and polyamine levels among cultured skin fibroblasts and hBMSC. Cultured skin fibroblasts and hBMSC had comparable steady state SMS mRNA levels (Figure 3B) and SMS protein levels (data not shown), suggesting that differential SMS expression is an unlikely basis for expression of SRS features. Additionally, excluding differential degradation of the mutant SMS protein as the basis for expression of SRS features, the cultured hBMSC derived from Patient II-1 had SMS protein levels only 1.7-fold lower than for control hBMSC (Figure 3C-K), whereas his fibroblasts had 2.6-fold less SMS protein compared to control fibroblasts.

To test if differences in polyamine metabolism might contribute to the differential expression of SRS features, we compared polyamine levels in cultured skin fibroblasts and hBMSCs. Skin fibroblast lysates derived from Patients II-1 and II-3 had mean spermidine levels 1.78fold higher than control fibroblasts $(p=0.28)$ and mean spermine levels 7.40-fold lower than control fibroblasts $(\mathrm{p}=0.21)$. In contrast, lysates of cultured hBMSCs from Patient II-1 contained mean spermidine levels 5.07-fold higher $(\mathrm{SEM}=22.8, \mathrm{p}=0.001)$ than control hBMSCs (Figure $3 \mathrm{~L}$ ) and had no detectable spermine.

\section{SRS hBMSCs have decreased osteogenic activity}

To determine the differences in osteogenic potential, hBMSCs isolated from an unaffected control and Patient II-1 were treated with osteogenic differentiation media and stained with Alizarin Red S, an anthraquinone dye that stains the calcium deposits indicative of mature osteocytes $[50,51]$. Based on the intensity of Alizarin Red S

Table 3 Calcium phosphate levels in patients II-1 and II-3

\begin{tabular}{llll}
\hline Serum Metabolite & Patient II-1 & Patient II-3 & Reference range \\
\hline Calcium (mg/dL) & $7.8-10.6$ & $6.6-10.1$ & $8.78-10.5$ \\
Phosphorus (mg/dL) & $2.4-5.1$ & $3.5-5.3$ & $3.1-5.1$ \\
\hline
\end{tabular}


staining, the differentiated hBMSCs from Patient II-1 produced markedly fewer calcium deposits than did those from the control (Figure 3M). Addition of $1 \mu \mathrm{M}$ spermine did not alter the Alizarin Red S staining (data not shown).

\section{Discussion}

We report two brothers with SRS in whom we identified a SMS mutation (NM_004595.4:c.443A > G, p.Gln148Arg) resulting in near absence of enzyme activity and decreased steady state SMS protein levels. To better delineate the tissue-specificity of SRS features, we investigated the low bone density of SMS and observed functional osteoblast and osteoclast deficiencies, a marked spermidine and spermine imbalance in hBMSCs and poor calcium phosphate mineralization by differentiated hBMSCs. We therefore speculate that polyamines play a critical role in osteoblasts that is not required by other cells, such as lymphoblasts and fibroblasts.

The p.Gln148Arg mutation represents the first SMS mutation to alter the MTA binding site [52]. Disturbance of MTA homeostasis could contribute to the more severe phenotype of the propositi relative to that of other SRS patients. MTA is needed for the transfer of the aminopropyl group from decarboxylated S-adenosylmethionine (dcAdoMet) to spermidine; it is also an inhibitor of SRM and SMS [53], and consequently decreases cellular spermine concentrations [54]. Indeed, the spermine:spermidine ratio of our patients' cells was reduced 3-fold more than the ratio for cells tested from any other SRS patient (C.S., unpublished data).

The severity of the phenotype in the propositi relative to other individuals with SRS might alternatively be attributable to differing insults or genetic backgrounds. For example, the perinatal intraventricular hemorrhage of patient II-1 might contribute to the severity of his neurological features, although the presence of similar neurological features in his brother, who did not have an ischemic or hemorrhagic brain insult, suggests this is not a substantial contributor. On the other hand, the variant in FRMPD4 might modify the genetic background and thereby contribute to the intellectual disability of the propositi. We did not identify other environmental insults or appropriately segregating, pathogenic variants to explain the additional features of the propositi. Consequently, if other genetic contributors have a significant role in modifying the phenotype of SRS, they were either not detected by our exome sequencing or were common polymorphisms excluded by our analyses. It remains possible that epigenetic and stochastic factors also modulate the expressivity of SRS.

Central neuroendocrine signaling does not appear to be impaired in SRS; however, peripheral neuroendocrine signaling including hypogonadism and altered calcium and phosphate homeostasis might contribute as might other issues such as immobilization, renal tubular acidosis, low muscle mass and medications. It is thought that male hypogonadism decreases bone mineral density because androgens promote osteoblast differentiation and proliferation and decrease the activity of osteoclasts [55]. Additionally, impaired calcium and phosphate homeostasis impede osteoclast function [56], and hypercalciuria is associated with decreased bone density, as well as the nephrocalcinosis seen in this patient. Several anticonvulsants, including clonazepam and topiramate, are also associated with decreases in bone mineral density [57]. These factors alone are unlikely to fully account for the decreased bone mineral density observed in our patients, since other SRS individuals have had low bone density in the absence of these issues [11]. Rather, the predominant mechanism of osteoporosis in SRS is likely related to impaired polyamine metabolism.

The disease mechanisms and phenotypic expansion reported herein provide some insight for the management of SRS. Optimal control of the metabolic abnormalities, limited use of medications known to affect bone, and appropriate physical therapy should be part of the management plan in any chronically ill, immobilized individual. Since analysis of the bone did not detect increased osteoclastic activity, bisphosphonate therapy would likely be of minimal effectiveness unless further studies refute our observations. In addition, if study of additional patients establishes the association of renal disease, retinal pigmentary changes, and perturbations of glucose homeostasis with SRS, then screening for and symptomatic management of these problems has the potential to improve patient care.

\section{Conclusions}

This report identifies a novel SRS-associated SMS mutation, p. Gln148Arg, and expands the SRS phenotype. It also provides the first evidence that SRS patients have a loss of osteoblast and osteoclast activity and that the low bone density of SRS likely arises by a cell intrinsic process.

\section{Consent}

The patients' parents gave written, informed consent for publication of this case report and any accompanying photographs.

\section{Additional file}

Additional file 1: Table S1. CNVs detected in the propositi. Table S2. Exome variants meeting rarity and predicted deleteriousness requirements and segregating with disease. Figure S1. Expression profile of SMS mRNA and protein. (A) Graph showing qRT-PCR detection of SMS mRNA levels in total RNA extracted from the respective tissues. SMS mRNA levels were normalized to the mRNA levels of GAPDH. (B) Immunoblot showing SMS protein levels in lysates from the respective tissues. (C) SMS protein expression in each tissue plotted relative to GAPDH protein expression. 


\section{Competing interests}

The authors declare that they have no competing interests.

\section{Authors' contributions}

JSA designed, performed and interpreted all of thepolyamine assays, the protein and RNA expression and imaging assays and drafted the manuscript. Coordinated the re-admission of the patient so, bone biopsy and further endocrinology studies could be obtained. NB cultured human bone marrow stromal cells and performed the differentiation experiments and the calcification assays. LW was the primary nurse practitioner involved with thepatients. She coordinated the clinical sample testing and performed history and physical exam. WB aided in the culturing human bone marrow stromal cells and performed the differentiation experiments and the calcification assay and aided in the analysis of the NGS and SNP chip data. VM cultured human bone marrow stromal cells and performed the differentiation experiments and the calcification assays. JA did the sanger confirmation. DA was the attending physician when the patient was admitted, and coordinated clinical care while at the NIH. CS helped with coordination of the enzyme assay and provided samples from other individuals with SRS and expertise about the disorder. JN performed the spermine synthase enzymatic assay. TW helped with interpretation of the enzymatic assays. RG Was the endocrinologist involved with the case that helped partition the bone biopsy sample in the operating room and coordinated bone marrow stem cell culture. MC provided consultation for endocrinology findings. LT performed the bone biopsy. TM performed the NGS analysis that identified the variant in SMS. WG coordinated the admission of these individuals into the UDP, curated the chart, provided insights into interpretation of data and obtained funding for the studies. All authors read and approved the final manuscript.

\section{Acknowledgements}

We thank Drs. Paul Lee, May Christine Malicdan and Grace Zhai for critical review of the manuscript. We would like to thank Dr. Edward McCarthy at The Johns Hopkins School of Medicine for performing the bone histomorphometry, Dr. Camilo Toro for imaging interpretations and Shira Ziegler for technical guidance for the alkaline phosphatase assay. This work was supported by the Intramural Research Programs of the National Human Genome Research Institute and National Institute of Dental and Craniofacial Research and the Common Fund of the NIH Office of the Director, National Institutes of Health. This work as also supported by extramural funding from NINDS (NS073854) and South Carolina Department of Disabilities and Special Needs (SCDDSN).

\section{Author details}

${ }^{1}$ Undiagnosed Diseases Program, Common Fund, Office of the Director, National Institutes of Health, Bethesda, MD 20814, USA. ${ }^{2}$ Medical Genetics Branch, National Human Genome Research Institute, Bethesda, MD, USA. ${ }^{3}$ Skeletal Clinical Studies Unit, Craniofacial and Skeletal Disease Branch, National Institute of Dental and Craniofacial Research, National Institutes of Health, Bethesda, MD 20892, USA. ${ }^{4}$ J.C. Self Research Institute, Greenwood Genetics Centre, Greenwood, SC 29646, USA. ${ }^{5}$ George Washington University School of Medicine, Washington, DC, USA. ${ }^{6}$ Children's National Medical Center, Washington, DC, USA.

Received: 9 July 2014 Accepted: 28 January 2015

Published online: 07 March 2015

\section{References}

1. D'Agostino L, Di Luccia A. Polyamines interact with DNA as molecular aggregates. Eur J Biochem. 2002;269(17):4317-25.

2. Watanabe S, Ksama-Equchi K, Kobayashi H, Igarashi K. Estimation of polyamine binding to macromolecules and ATP in bovine lymphocytes and rat liver. J Biol Chem. 1991;266(31):20803-9.

3. Minois N, Carmona-Gutierrez D, Madeo F. Polyamines in aging and disease. Aging. 2011;3(8):716-32.

4. Raina A, Janne J. Physiology of the natural polyamines putrescine, spermidine and spermine. Med Biol. 1975;53(3):121-47.

5. Pegg AE, Michael AJ. Spermine synthase. Cell Mol Life Sci. 2010;67(1):113-21.

6. Williams K. Interactions of polyamines with ion channels. Biochem J. 1997;325(Pt 2):289-97.
7. Janne O, Bardin CW, Jacob ST. DNA-dependent RNA polymerases I and II from kidney. Effect of polyamines on the in vitro transcription of DNA and chromatin. Biochemistry. 1975;14(16):3589-97.

8. Friedman ME, Bachrach $U$. Inhibition of protein synthesis by spermine in growing cells of Staphylococcus aureus. J Bacteriol. 1966;92(1):49-55.

9. Nichols CG, Lopatin AN. Inward rectifier potassium channels. Annu Rev Physiol. 1997;59:171-91.

10. Snyder RD, Robinson A. Recessive sex-linked mental retardation in the absence of other recognizable abnormalities. Report of a family. Clin Pediatr. 1969;8(11):669-74.

11. Arena JF, Schwartz C, Ouzts L, Stevenson R, Miller M, Garza J, et al. X-linked mental retardation with thin habitus, osteoporosis, and kyphoscoliosis: linkage to Xp21.3-p22.12. Am J Med Genet. 1996;64(1):50-8.

12. Cason AL, Ikequchi Y, Skinner C, Wood TC, Holder KR, Lubs JA, et al. X-linked spermine synthase gene (SMS) defect: the first polyamine deficiency syndrome. Eur J Hum Genet. 2003;11(12):937-44.

13. de Alencastro G, McCloskey DE, Kliemann SE, Maranduba CM, Pegg AE, Wang $X$, et al. New SMS mutation leads to a striking reduction in spermine synthase protein function and a severe form of Snyder-Robinson X-linked recessive mental retardation syndrome. J Med Genet. 2008;45(8):539-43.

14. Becerra-Solano LE, Butler J, Castaneda-Cisneros G, McCloskey DE, Wang X, Pegg AE, et al. A missense mutation, p.V132G, in the X-linked spermine synthase gene (SMS) causes Snyder-Robinson syndrome. Am J Med Genet. 2009;149A(3):328-35.

15. Schwartz CE, Wang X, Stevenson RE, Pegg AE. Spermine Synthase Deficiency Resulting in X-Linked Intellectual Disability (Snyder-Robinson Syndrome). In: Pegg AE, Castero Jr RA, editors. Polyamines: Methods and Protocols. New York: Humana Press; 2011. p. 437-45.

16. Peron A, Spaccini L, Norris J, Bova SM, Selicorni A, Weber G, et al. Snyder-Robinson syndrome: a novel nonsense mutation in spermine synthase and expansion of the phenotype. Am J Med Genet A. 2013;161(9):2316-20.

17. Zhang Z, Norris J, Kalscheuer V, Wood T, Wang L, Schwartz C, et al. A Y328C missense mutation in spermine synthase causes a mild form of Snyder-Robinson syndrome. Hum Mol Genet. 2013;22(18):3789-97.

18. Raisz LG. Pathogenesis of osteoporosis: concepts, conflicts, and prospects. J Clin Invest. 2005;115(12):3318-25.

19. Guntur AR, Rosen CJ. Bone as an endocrine organ. Endocr Pract. 2012;18(5):758-62.

20. Albert J, Schwartz CE, Boerkoel CF, Stevenson RE. Snyder-Robinson Syndrome In: Pagon RA, Adam MP, Ardinger HH, Bird TD, Dolan CR, Fong $C T$, et al., editors. Seattle (WA): GeneReviews(R); 1993.

21. Zemel BS, Leonard MB, Kelly A, Lappe JM, Gilsanz V, Oberfield S, et al. Height adjustment in assessing dual energy $\mathrm{x}$-ray absorptiometry measurements of bone mass and density in children. J Clin Endocrinol Metab. 2010;95(3):1265-73.

22. Wang K, Li M, Hadley D, Liu R, Glessner J, Grant SF, et al. PennCNV: an integrated hidden Markov model designed for high-resolution copy number variation detection in whole-genome SNP genotyping data. Genome Res. 2007;17(11):1665-74.

23. Manoli I, Golas G, Westbroek W, Vilboux T, Markello TC, Introne W, et al. Chediak-Higashi syndrome with early developmental delay resulting from paternal heterodisomy of chromosome 1. Am J Med Genet A. 2010;152A(6):1474-83.

24. Markello TC, Adams DR, Genome-scale sequencing to identify genes involved in Mendelian disorders. Current protocols in human genetics / editorial board, Jonathan L. Haines... [et al], 2013. 79: p. Unit 613.

25. Adams DR, Sincan M, Fuentes Fajardo K, Mullikin JC, Pierson TM, et al. Analysis of DNA sequence variants detected by highthroughput sequencing. Hum Mutat. 2012;33(4):599-608.

26. Markello TC, Han T, Carlson-Donohoe H, Ahaghotu C, Harper U, Jones M, et al. Recombination mapping using Boolean logic and highdensity SNP genotyping for exome sequence filtering. Mol Genet Metab. 2012;105(3):382-9.

27. Adzhubei IA, Schmidt S, Peshkin L, Ramensky VE, Gerasimova A, Bork P, et al. A method and server for predicting damaging missense mutations. Nat Methods. 2010;7(4):248-9.

28. Johnston JJ, Teer JK, Cherukuri PF, Hansen NF, Loftus SK, NIH Intramural Sequencing Center (NISC), et al. Massively parallel sequencing of exons on the $\mathrm{X}$ chromosome identifies RBM10 as the gene that causes a syndromic form of cleft palate. Am J Hum Genet. 2010;86(5):743-8. 
29. Kumar P, Henikoff S, Ng PC. Predicting the effects of coding nonsynonymous variants on protein function using the SIFT algorithm. Nat Protoc. 2009;4(7):1073-81.

30. Ng PC, Henikoff S. Predicting the effects of amino acid substitutions on protein function. Annu Rev Genomics Hum Genet. 2006;7:61-80.

31. Ng PC, Henikoff S. SIFT: Predicting amino acid changes that affect protein function. Nucleic Acids Res. 2003;31(13):3812-4.

32. Ng PC, Henikoff S. Accounting for human polymorphisms predicted to affect protein function. Genome Res. 2002;12(3):436-46.

33. Ng PC, Henikoff S. Predicting deleterious amino acid substitutions. Genome Res. 2001;11(5):863-74.

34. Henikoff S, Henikoff JG. Amino acid substitution matrices from protein blocks. Proc Natl Acad Sci U S A. 1992;89(22):10915-9.

35. McCarthy EF, Earnest K, Rossiter K, Shapiro J. Bone histomorphometry in adults with type IA osteogenesis imperfecta. Clin Orthop Relat Res. 1997;336:254-62.

36. Sowell J, Norris J, Jones K, Schwartz C, Wood T. Diagnostic screening for spermine synthase deficiency by liquid chromatography tandem mass spectrometry. Clin Chim Acta. 2011;412(7-8):655-60.

37. Bhattacharyya N, Wiench M, Dumitrescu C, Connolly BM, Bugge TH, Patel $\mathrm{HV}$, et al. Mechanism of FGF23 processing in fibrous dysplasia. J Bone Miner Res Off J Am Soc Bone Miner Res. 2012;27(5):1132-41.

38. Kodiha M, Umar R, Stochaj U. Optimized immunofluorescence staining protocol to detect the nucleoporin Nup98 in different subcellular compartments. Protocol Exchange, 2009.

39. Wu H, Min J, Zeng H, McCloskey DE, Ikeguchi Y, Loppnau P, et al. Crystal structure of human spermine synthase: implications of substrate binding and catalytic mechanism. J Biol Chem. 2008;283(23):16135-46.

40. Schrodinger, LLC. The PyMOL Molecular Graphics System, Version 1.5.0.5, 2010.

41. Piton A, Gauthier J, Hamdan FF, Lafreniere RG, Yang Y, Henrion E, et al. Systematic resequencing of $X$-chromosome synaptic genes in autism spectrum disorder and schizophrenia. Mol Psychiatry. 2011;16(8):867-80.

42. Lee HW, Choi J, Shin H, Kim K, Yang J, Na M, et al. Preso, a nove PSD-95-interacting FERM and PDZ domain protein that regulates dendritic spine morphogenesis. J Neurosci Off J Soc Neurosci. 2008;28(53):14546-56.

43. Nagao M, Feinstein TN, Ezura Y, Hayata T, Notomi T, Saita Y, et al. Sympathetic control of bone mass regulated by osteopontin. Proc Natl Acad Sci U S A. 2011;108(43):17767-72.

44. Kajimura D, Hinoi E, Ferron M, Kode A, Riley KJ, Zhou B, et al. Genetic determination of the cellular basis of the sympathetic regulation of bone mass accrual. J Exp Med. 2011;208(4):841-51.

45. Takeda S, Karsenty G. Molecular bases of the sympathetic regulation of bone mass. Bone. 2008:42(5):837-40.

46. Takeda S, Elefteriou F, Levasseur R, Liu X, Zhao L, Parker KL, et al. Leptin regulates bone formation via the sympathetic nervous system. Cell. 2002;111(3):305-17.

47. Elefteriou F, Ahn JD, Takeda S, Starbuck M, Yang X, Liu X, et al. Leptin regulation of bone resorption by the sympathetic nervous system and CART. Nature. 2005;434(7032):514-20.

48. Kondo H, Nifuji A, Takeda S, Ezura Y, Rittling SR, Denhardt DT, et al. Unloading induces osteoblastic cell suppression and osteoclastic cell activation to lead to bone loss via sympathetic nervous system. J Biol Chem. 2005;280(34):30192-200.

49. Fu L, Patel MS, Bradley A, Wagner EF, Karsenty G. The molecular clock mediates leptin-regulated bone formation. Cell. 2005;122(5):803-15.

50. Jackson $L$, Jones $D R$, Scotting $P$, Sottile $V$. Adult mesenchymal stem cells: differentiation potential and therapeutic applications. J Postgrad Med. 2007:53(2):121-7.

51. Sottile V, Halleux C, Bassilana F, Keller H, Seuwen K. Stem cell characteristics of human trabecular bone-derived cells. Bone. 2002;30(5):699-704.

52. Ikeguchi $Y$, Bewley MC, Pegg AE. Aminopropyltransferases: function, structure and genetics. J Biochem. 2006;139(1):1-9.

53. Williams-Ashman HG, Seidenfeld J, Galletti P. Trends in the biochemical pharmacology of 5'-deoxy-5'-methylthioadenosine. Biochem Pharmacol. 1982;31(3):277-88
54. Raina A, Tuomi K, Pajula RL. Inhibition of the synthesis of polyamines and macromolecules by 5'-methylthioadenosine and 5'-alkylthiotubercidins in BHK21 cells. Biochem J. 1982;204(3):697-703.

55. Dupree K, Dobs A. Osteopenia and male hypogonadism. Rev Urol. 2004;6 Suppl 6:S30-4.

56. Ross AC. The 2011 report on dietary reference intakes for calcium and vitamin D. Public Health Nutr. 2011;14(5):938-9.

57. Lee RH, Lyles KW, Colon-Emeric C. A review of the effect of anticonvulsant medications on bone mineral density and fracture risk. Am J Geriatr Pharmacother. 2010;8(1):34-46.

\section{Submit your next manuscript to BioMed Central and take full advantage of:}

- Convenient online submission

- Thorough peer review

- No space constraints or color figure charges

- Immediate publication on acceptance

- Inclusion in PubMed, CAS, Scopus and Google Scholar

- Research which is freely available for redistribution 